\title{
Stability Analysis for Grain Yield and Quality Parameters in QPM (Zea mays L.) Inbred Line Crosses
}

\author{
Baudh Bharti $^{1^{*}}$, R.B. Dubey, Arun Kumar ${ }^{2}$, Amit Dadheech and Rohit Kumar Dhobi ${ }^{3}$ \\ ${ }^{1}$ Department of Plant Breeding and Genetics, ${ }^{3}$ Department of Horticulture, Maharana Pratap \\ University of Agriculture and Technology, Udaipur-313001, Rajasthan, India \\ ${ }^{2}$ Department of Genetics and Plant Breeding, Govind Ballabh Pant University of Agriculture \& \\ Technology, Pantnagar-263145, Uttarakhand, India \\ *Corresponding author
}

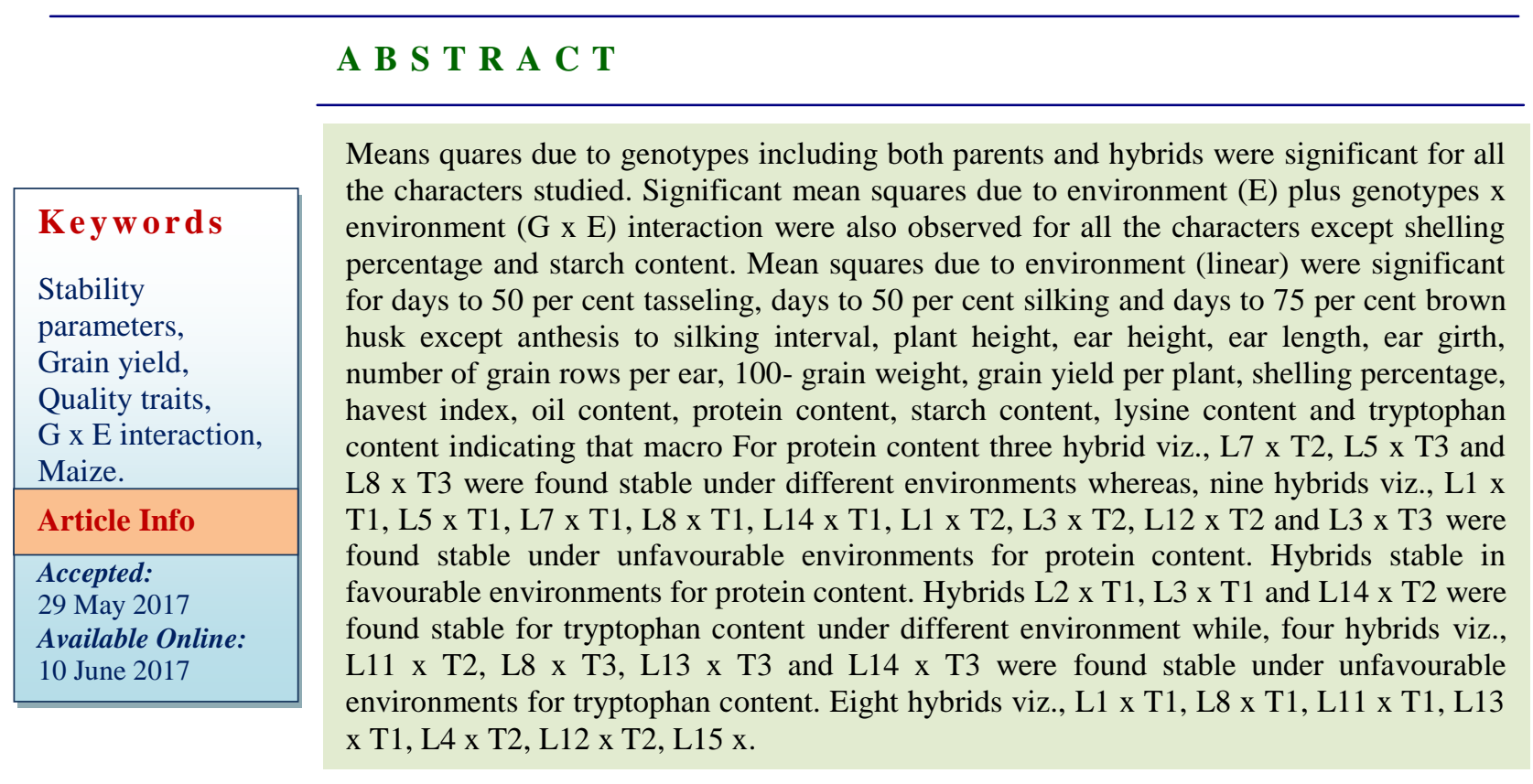

\section{Introduction}

Maize (Zea mays L.) $2 \mathrm{n}=20$, is the third most important cereal crop after rice and wheat in the world. It is referred to as "Miracle Crop" and "Queen of the Cereals" due to its high productivity potential compared to other Graminae family members. It is believed to have originated in Southern Mexico or Northern Guatemala (Weather wax, 1955). Protein of maize is deficient in two essential amino acids, lysine and tryptophan (Bhatia and Rabson, 1987). In non-QPM, the endosperm contains a high amount of zein which is completely devoid of lysine and tryptophan.

Maize is used as human food, chemicals, medicines, biofuel, ornamental and other uses e. g. variegated and coloured leaf forms as well as those with colourful ears are used (Wikipedia, 2011). The poor nutritional value 
of normal maize grain has been well known for a long time (Osborne and Mendel, 1914). Maize has protein content with protein quality limited by deficiencies of two essential amino acids like lysine and tryptophan and has excess of luecine and gsoleucine, leading to a poor growth in children and pellagra in adults (Mertz et al., 1964). Maize protein is characterized by high level of glutamic acid and leucine. Lysine (1.2\% of protein) and tryptophan $(0.4 \%$ of protein) are limiting amino acid in maize. This is due to the fact that major storage protein is a prolamin fraction zein, which forms up to 50 to $60 \%$ of the storage protein. Zein consists of a group of hydrophobic proteins, completely devoid of lysine and tryptophan (Inglett, 1970). Hence, genetic manipulation for improved nutritional value, particularly protein quality was considered as a noble goal. This effort was stimulated by the 1963 discovery of mutant maize called as "opaque-2 gene".

The lysine levels in normal and quality protein maize (QPM) average $2.0 \%$ and $4.0 \%$ of total protein respectively, but range across genetic background from 1.6 to $2.6 \%$ in normal maize and 2.7 to $4.5 \%$ in their opaque-2 maize converted counter parts (Moro et al., 1996). The lysine content of quality protein maize (QPM) in whole grains range from 0.33 to 0.54 per cent, with the average of 0.38 per cent and 46 per cent higher than normal maize. The tryptophan content is 0.08 per cent, which is 6.6 per cent higher than normal maize (Ortega et al., 1986, Sproule et al., 1988, Osei et al., 1999).

\section{Materials and Methods}

The experimental material was generated by making crosses between 15 inbred lines and 3 testers in line $x$ tester mating design. Fifteen parental inbred lines were crossed with three testers during rabi 2013 to generate the experimental hybrids for this study. The experimental material, thus, consisting of 45 F1s (single crosses), 15 inbred lines, 3 testers and 4 checks (HQPM-1, HQPM-5, Pratap QPM-1 and Vivek QPM-9) were evaluated during kharif and rabi 2014 in the three environments. Three environments were created by two locations and date of sowing viz., E1 (timly sowning, kharif 2014 at Instructional farm Rajasthan college of Agriculture, Udaipur), E2 (timly sowning, kharif 2014 at ARSS, Vallabh Nagar, MPUAT, Udaipur) and E3 (timly sowning, rabi 2014-15 at Instructional farm Rajasthan college of Agriculture, Udaipur). The experimental material was planted under each environment in randomized bock design with three replication in a single row plot of four meter length, maintaining crop geometry of $60 \times 25 \mathrm{~cm}$. All the recommended agronomy inputs and practices were applied to the crop during the season, to raise the successful crop. The NPK fertilizer were applied at the rate of 120:60:00 $\mathrm{kg} / \mathrm{ha}$. The total amount of phosphatic fertilizer and half of the nitrogenous fertilizer were applied as basal dose and rest of the nitrogenous fertilizer were given in two equal doses, one at kneehigh stage and another at flowering stage of the crop. The border rows were also planted to neutralize the border effect. The mean values were used for statistical analysis. Stability analysis was done using the model ofEberhart and Russel (1966).

\section{Results and Discussion}

The analysis of variance for phenotypic stability (Table 1) revealed that mean squares due to genotypes including both parents and hybrids were significant for all the characters studied. Significant mean squares due to environment (E) plus genotypes $x$ environment $(\mathrm{G} \times \mathrm{E})$ interaction were also observed for all the characters except shelling percentage and starch content. Mean squares due to environment (linear) were significant 
for days to 50 per cent tasseling, days to 50 per cent silking and days to 75 per cent brown husk except anthesis to silking interval, plant height, ear height, ear length, ear girth, number of grain rows per ear, 100- grain weight, grain yield per plant, shelling percentage, havest index, oil content, protein content, starch content, lysine content and tryptophan content indicating that macro environmental differences were present under all the three environments studies.

The mean of squares due to genotypes $x$ environment interactions were also significant for all the characters except starch content. Indicating the influence of environmental conditions on the genotypes evaluated.

The significant $G \times E$ interaction for various traits were also reported by Sharma and Saikia (2000), Dodiya and Joshi (2003), Singh et al., (2003), Abera et al., (2006), Admassu et al., (2008), Zaidi et al., (2008), Rahman et al., (2010), Arulselvi and Selvi (2010), Beyene et al., (2011), Shiri (2013), Nzuve et al., (2013), Kamutando et al., (2013) and Sserumaga et al., (2016).A perusal of stability parameters for grain yield per plant revealed that out of 67 genotypes 59 genotypes (18 parents, 37 hybrids and 4 checks) exhibited non-significant deviation from regression (S2di) and are as such predictable for this trait. Parental line L6 exhibited non-significant deviation from regression (S2di) and regression coefficient greater than unity (bi >1) with higher mean values than the population mean and would remain stable for grain yield per plant in favourable environments. Twenty six hybrids viz., L1 X T1, L4 X T1, L5 X T1, L6 x T1, L8 x T1, L9 x T1, L10 x T1, L12 x T1, L14 x T1, L15 x T1, L2 x T2, L4 x T2, L5 x T2, L6 xT2, L7 x T2, L14 x T2, L3 x T3, L4 x T3, L5 x T3, L6 x T3, L8 x T3, L10 x T3, L11 x T3, L12 x T3, L14 x T3 and L15 x T3 and two checks namely HQPM-1 and Vivek
QPM-9 exhibited non-significant deviation from regression (S2di) and regression coefficient greater than unity (bi >1) with higher mean values than the population mean and thereby indicating their stability under favourable environments. Hybrids L2 x T1 and L3 x T1 exhibited non-significant S2di and regression coefficient nearly equal to unity ( $\mathrm{bi}=1$ ) with higher mean values than the population mean, thereby indicating stability under different environments for grain yield per plant. Three other hybrids viz., L9 x T2, L12 x T2 and L15 x T2 and check HQPM-5 exhibited non-significant deviation from regression (S2di) and regression coefficient less than unity (bi <1) with higher mean values than the population mean, thereby indicating their suitability and stability under unfavourable environments. Out of 67 genotypes, 8 genotypes (2 parents and 6 hybrids) exhibited non-significant deviation from regression (S2di), indicating their predictable behaviour for oil content.

Parental line L12 exhibited non-significant deviation from regression (S2di) and regression coefficient greater than unity (bi $>1$ ) with higher mean values than the population mean. This line thus showed its superiority and stability under favourable environments. Two hybrids viz., L14 x T3 and L15 $x \quad$ T3 showed non-significant deviation from regression (S2di) and regression coefficient less than unity (bi <1) with higher mean values than the population mean were as such considered stable and suitable under unfavourable environments for oil content. Hybrid L6 x T3 exhibited nonsignificant deviation from regression (S2di) and regression coefficient nearly equal to unity (bi =1) with higher mean values than the population mean, thereby indicating stability under different environments. Hybrid L9 x T3 exhibited non-significant deviation from regression (S2di) and regression coefficient greater than unity (bi >1) with higher mean 
values than the population mean, thereby indicating stability under favourable environments.

Out of 67 genotypes, 65 genotypes showed non-significant deviation from regression (S2di) indicating their predictable behaviour.

Parents L6 and T1 exhibited non- significant S2di and regression coefficient nearly equal to unity (bi =1) with higher mean values than the population mean, thereby indicating stability under different environments for protein content. Two other parents viz., L10 and L13 exhibited non-significant deviation from regression (S2di) and regression coefficient greater than unity (bi >1) with higher mean values than the population mean. These parents thus showed its superiority and stability under favourable environments.

The present study showed the improvement of sperm quality during preservation at $5^{\circ} \mathrm{C}$ Nine hybrids viz., L1 x T1, L5 x T1, L7 x T1, L8 x T1, L14 x T1, L1 x T2, L3 x T2, L12 x T2 and L3 $x \quad$ T3 exhibited non-significant deviation from regression (S2di) and regression coefficient less than unity (bi $<1$ ) and higher mean values as compared to the population mean, were considered suitable and stable under unfavourable environments. Three hybrids viz., L7 x T2, L5 x T3 and L8 x T3 exhibited non-significant deviation from regression (S2di) and regression coefficient nearly equal to unity $(\mathrm{bi}=1)$ with higher mean values as compared to the population mean. These hybrids were found stable in different environments. Eleven other hybrids viz., L4 x T1, L10 x T1, L11 x T1, L6 x T2, L14 x T2, L1 x T3, L9 x T3, L11 x T3, L13 x T3, L14 x $\mathrm{T} 3$ and L15 x T3 exhibited non-significant deviation from regression (S2di) and regression coefficient greater than unity (bi $>1$ ) with higher mean values than the population mean.

Table.1 Analysis of variance Eberhart and Russel (1966) for grain yield and Quality traits in quality protein maize

\begin{tabular}{|c|c|c|c|c|c|c|c|}
\hline \multirow[t]{2}{*}{$\mathrm{SN}$} & Characters & Genotype & $\mathrm{E}+(\mathrm{G} \times \mathrm{E})$ & $\mathrm{E}(\mathrm{L})$ & $\mathrm{G} \times \mathrm{E}(\mathrm{L})$ & $\begin{array}{l}\text { Pool } \\
\text { dev. }\end{array}$ & $\begin{array}{c}\text { Pool } \\
\text { Err }\end{array}$ \\
\hline & & [66] & [134] & [1] & [66] & [67] & [396] \\
\hline 1 & $\begin{array}{l}\text { Grain yield per plant } \\
\text { (g) }\end{array}$ & $1275.04 * *$ & $102.81 * *$ & 2.19 & $194.51 * *$ & $13.99 * *$ & 9.20 \\
\hline 2 & Oil content $\%$ & $0.25^{* *}$ & $0.51 * *$ & 0.00 & $0.65^{* *}$ & $0.39 * *$ & 0.00 \\
\hline 3 & Protein content $\%$ & $2.65 * *$ & $0.01 * *$ & 0.00 & $0.02 * *$ & 0.00 & 0.01 \\
\hline 4 & Starch content $\%$ & $35.21 * *$ & 0.01 & 0.00 & 0.02 & 0.00 & 0.17 \\
\hline 5 & Lysine content \% & $0.82 * *$ & $0.00 * *$ & 0.00 & $0.00 * *$ & $0.00 * *$ & 0.00 \\
\hline 6 & $\begin{array}{l}\text { Tryptophan content } \\
\%\end{array}$ & $0.03 * *$ & $0.00 * *$ & 0.00 & $0.00 * *$ & 0.00 & 0.00 \\
\hline
\end{tabular}

*,** Significant at 5 and 1 per cent respectively. 
Table.2 Stability parameters for grain yield and quality traits in quality protein maize

\begin{tabular}{|c|c|c|c|c|c|c|c|c|c|c|}
\hline \multirow[t]{2}{*}{$\mathrm{SN}$} & \multirow[t]{2}{*}{ Genotype } & \multicolumn{3}{|c|}{ Grain Yield Per Plant $(\mathrm{g})$} & \multicolumn{3}{|c|}{ Oil content $\%$} & \multicolumn{3}{|c|}{ Protein content $\%$} \\
\hline & & $\mu$ & $\mathrm{b}_{\mathrm{i}}$ & $\mathrm{S}^{2} \mathrm{~d}_{\mathrm{i}}$ & $\mu$ & $b_{i}$ & $\mathrm{~S}^{2} \mathrm{~d}_{\mathrm{i}}$ & $\mu$ & $b_{i}$ & $\mathrm{~S}^{2} \mathrm{~d}_{\mathrm{i}}$ \\
\hline 1 & $\mathrm{~T} 1$ & 52.89 & $0.69 *$ & -8.975 & 3.81 & 2.42 & $0.438 * *$ & 9.46 & 0.92 & 0.002 \\
\hline 2 & $\mathrm{~T} 2$ & 60.20 & 0.53 & -3.105 & 4.12 & 2.07 & $0.448 * *$ & 7.72 & 1.08 & 0.009 \\
\hline 3 & $\mathrm{~T} 3$ & 67.48 & $0.58 * *$ & -9.190 & 4.18 & $-5.01 *$ & $0.004 *$ & 7.77 & 0.63 & 0.000 \\
\hline 4 & L1 & 37.36 & 0.34 & -7.029 & 4.09 & -2.28 & $0.013 * *$ & 8.16 & 1.34 & -0.001 \\
\hline 5 & L2 & 40.76 & 0.24 & -2.077 & 4.17 & 1.47 & $0.487 * *$ & 8.67 & 1.07 & -0.005 \\
\hline 6 & L3 & 47.82 & $0.38 *$ & -9.114 & 3.87 & -0.24 & $0.302 * *$ & 8.31 & 1.35 & -0.001 \\
\hline 7 & L4 & 44.53 & 0.48 & -8.905 & 3.81 & -2.30 & $0.550 * *$ & 7.73 & 1.01 & -0.004 \\
\hline 8 & L5 & 38.58 & 0.39 & -8.779 & 3.93 & 2.56 & $0.406 * *$ & 7.54 & -0.51 & -0.005 \\
\hline 9 & L6 & 76.22 & 1.12 & 9.600 & 4.34 & 4.44 & $0.284^{* *}$ & 8.94 & 0.96 & -0.003 \\
\hline 10 & L7 & 31.38 & 0.22 & 3.333 & 3.93 & 2.79 & $0.626 * *$ & 7.95 & 1.78 & 0.001 \\
\hline 11 & L8 & 43.33 & 0.31 & 13.359 & 4.21 & -3.49 & $1.643 * *$ & 8.24 & 1.23 & -0.004 \\
\hline 12 & L9 & 51.24 & $0.67 * *$ & -9.190 & 3.72 & -3.58 & $0.150 * *$ & 8.76 & 1.26 & -0.005 \\
\hline 13 & L10 & 36.89 & 0.25 & -1.779 & 4.30 & 3.12 & $0.145^{* *}$ & 9.82 & 1.01 & -0.003 \\
\hline 14 & L11 & 31.64 & $0.35 * *$ & -9.196 & 4.25 & 3.11 & $0.426^{* *}$ & 7.65 & 0.71 & -0.005 \\
\hline 15 & L12 & 29.02 & 0.17 & -8.902 & 4.27 & $7.53 *$ & 0.001 & 7.75 & -0.34 & -0.005 \\
\hline 16 & L13 & 39.66 & $0.42 *$ & -9.168 & 3.74 & -2.89 & $0.700 * *$ & 9.66 & 1.48 & -0.001 \\
\hline 17 & L14 & 46.96 & 0.44 & 14.491 & 4.09 & 2.69 & $0.553 * *$ & 8.61 & -0.48 & -0.005 \\
\hline 18 & L15 & 53.24 & 0.55 & -8.844 & 4.13 & $-1.52 *$ & -0.001 & 7.62 & 0.96 & 0.006 \\
\hline 19 & $\mathrm{~L} 1 \times \mathrm{T} 1$ & 84.51 & $1.35^{*}$ & -8.993 & 4.31 & 2.34 & $1.664 * *$ & 9.27 & 0.89 & -0.001 \\
\hline 20 & $\mathrm{~L} 2 \times \mathrm{T} 1$ & 88.42 & $0.99 *$ & -8.702 & 4.10 & 2.44 & $0.368 * *$ & 8.65 & 1.32 & -0.005 \\
\hline 21 & L3 x T1 & 82.27 & 0.93 & 24.003 & 4.41 & 4.35 & $0.472 * *$ & 8.51 & 0.87 & -0.002 \\
\hline 22 & $\mathrm{~L} 4 \times \mathrm{T} 1$ & 90.63 & $1.34 *$ & -8.991 & 4.32 & 3.28 & $0.103 * *$ & 9.27 & 1.32 & -0.004 \\
\hline 23 & L5 x T1 & 96.51 & 1.32 & 2.798 & 4.87 & -6.30 & $0.088 * *$ & 11.54 & 0.79 & -0.005 \\
\hline 24 & L6 x T1 & 96.96 & $1.42 *$ & -8.941 & 3.91 & 3.38 & $0.272^{* *}$ & 8.66 & 0.59 & -0.002 \\
\hline 25 & $\mathrm{~L} 7 \times \mathrm{T} 1$ & 72.91 & 0.68 & $118.945^{* *}$ & 4.23 & 5.05 & $0.286^{* *}$ & 10.36 & $-1.86 *$ & -0.005 \\
\hline 26 & L8 x T1 & 87.51 & 1.01 & 12.104 & 4.41 & -0.77 & $1.527 * *$ & 9.61 & $0.89 *$ & -0.005 \\
\hline 27 & L9 x T1 & 77.02 & $1.19 *$ & -8.574 & 4.19 & 6.81 & $0.732 * *$ & 8.20 & 0.29 & -0.005 \\
\hline 28 & $\mathrm{~L} 10 \times \mathrm{T} 1$ & 94.64 & $1.50 *$ & -8.429 & 4.35 & -0.52 & $0.760 * *$ & 9.63 & 1.08 & -0.003 \\
\hline 29 & $\mathrm{~L} 11 \times \mathrm{T} 1$ & 86.69 & 0.47 & $34.433 *$ & 4.14 & $-4.08 *$ & -0.001 & 8.94 & 1.77 & 0.005 \\
\hline 30 & $\mathrm{~L} 12 \times \mathrm{T} 1$ & 82.78 & $1.29 * *$ & -9.186 & 3.82 & 3.97 & 0.004 & 8.75 & 1.05 & -0.003 \\
\hline 31 & $\mathrm{~L} 13 \times \mathrm{T} 1$ & 68.44 & $0.99 *$ & -9.115 & 4.09 & -1.81 & $0.030 * *$ & 8.63 & 0.33 & -0.005 \\
\hline 32 & $\mathrm{~L} 14 \times \mathrm{T} 1$ & 99.87 & 1.77 & 11.202 & 4.79 & 7.77 & $0.053 * *$ & 9.19 & 0.82 & -0.005 \\
\hline 33 & L15 x T1 & 89.24 & 1.01 & 3.964 & 4.10 & -0.91 & $0.770 * *$ & 8.75 & 0.48 & -0.001 \\
\hline 34 & $\mathrm{~L} 1 \times \mathrm{T} 2$ & 68.51 & $0.95 *$ & -9.138 & 3.74 & -1.89 & $0.354 * *$ & 12.11 & 0.69 & 0.003 \\
\hline 35 & $\mathrm{~L} 2 \times \mathrm{T} 2$ & 94.40 & $1.30 *$ & -9.022 & 4.21 & 0.58 & $0.032 * *$ & 8.30 & -0.08 & -0.002 \\
\hline 36 & $\mathrm{~L} 3 \times \mathrm{T} 2$ & 83.89 & 0.60 & $48.232 *$ & 3.82 & -5.57 & $1.369 * *$ & 9.30 & 0.55 & -0.004 \\
\hline 37 & $\mathrm{~L} 4 \times \mathrm{T} 2$ & 89.47 & $1.03 * *$ & -9.175 & 4.04 & 0.62 & $0.474 * *$ & 8.41 & 0.50 & -0.005 \\
\hline 38 & L5 x T2 & 83.93 & $1.34 *$ & -9.061 & 4.73 & 3.02 & $1.053 * *$ & 7.12 & -0.43 & -0.003 \\
\hline 39 & L6 x T2 & 94.00 & $1.92 *$ & -5.877 & 4.51 & -1.27 & $0.097 * *$ & 8.87 & 1.69 & -0.002 \\
\hline 40 & $\mathrm{~L} 7 \times \mathrm{T} 2$ & 101.86 & $1.99 *$ & -8.285 & 4.61 & 1.73 & $1.677 * *$ & 9.91 & 0.96 & -0.005 \\
\hline 41 & $\mathrm{~L} 8 \times \mathrm{T} 2$ & 89.40 & 0.58 & $50.764 *$ & 4.13 & -0.68 & $0.259 * *$ & 8.98 & $2.04 *$ & -0.005 \\
\hline 42 & L9 x T2 & 81.33 & $0.84 *$ & -8.557 & 3.86 & 2.44 & $0.017 * *$ & 8.44 & 0.93 & -0.004 \\
\hline 43 & $\mathrm{~L} 10 \times \mathrm{T} 2$ & 65.98 & 0.47 & -8.575 & 4.65 & -0.78 & $0.349 * *$ & 8.75 & 1.44 & -0.005 \\
\hline 44 & $\mathrm{~L} 11 \times \mathrm{T} 2$ & 72.82 & 0.63 & -3.234 & 4.23 & -6.11 & $0.198 * *$ & 8.44 & 1.18 & -0.003 \\
\hline 45 & $\mathrm{~L} 12 \times \mathrm{T} 2$ & 91.31 & 0.40 & 20.219 & 3.71 & -1.70 & $0.325 * *$ & 9.73 & -1.47 & 0.000 \\
\hline 46 & $\mathrm{~L} 13 \times \mathrm{T} 2$ & 72.42 & 0.56 & -8.857 & 4.54 & -2.37 & $0.291 * *$ & 8.90 & 0.30 & $0.034 * *$ \\
\hline 47 & $\mathrm{~L} 14 \times \mathrm{T} 2$ & 91.94 & $1.98 *$ & -8.279 & 3.73 & -0.20 & $0.809 * *$ & 10.16 & 3.22 & -0.005 \\
\hline 48 & $\mathrm{~L} 15 \times \mathrm{T} 2$ & 91.11 & $0.73 * *$ & -9.179 & 4.75 & 1.99 & $0.095 * *$ & 8.42 & 0.79 & -0.003 \\
\hline 49 & $\mathrm{~L} 1 \times \mathrm{T} 3$ & 67.80 & 0.45 & -7.545 & 4.16 & -3.50 & $0.762 * *$ & 9.42 & 1.49 & -0.005 \\
\hline 50 & $\mathrm{~L} 2 \times \mathrm{T} 3$ & 95.33 & 2.03 & $26.109 *$ & 4.28 & 2.72 & $0.161 * *$ & 8.76 & 1.49 & -0.003 \\
\hline 51 & L3 $x$ T3 & 90.27 & 1.40 & -4.215 & 4.29 & 9.64 & $0.060 * *$ & 9.38 & -0.26 & -0.003 \\
\hline 52 & L4 x T3 & 88.64 & 1.02 & -7.551 & 4.28 & 6.09 & $0.440 * *$ & 8.96 & $1.84 *$ & -0.005 \\
\hline 53 & L5 x T3 & 88.09 & $1.70 *$ & -8.540 & 4.62 & 1.53 & $0.013 * *$ & 9.90 & 0.96 & -0.005 \\
\hline 54 & L6 x T3 & 85.20 & $1.37 *$ & -8.392 & 4.32 & 0.90 & -0.001 & 8.69 & 2.37 & 0.006 \\
\hline 55 & L7 x T3 & 74.10 & 1.65 & $63.646 * *$ & 4.07 & -3.50 & $1.309 * *$ & 7.74 & 1.58 & -0.005 \\
\hline 56 & L8 x T3 & 90.62 & 1.63 & -5.053 & 4.65 & -0.76 & $0.216^{* *}$ & 9.63 & 0.96 & -0.005 \\
\hline 57 & L9 $x$ T3 & 69.16 & 1.37 & $75.254 * *$ & 4.58 & $3.91 *$ & -0.001 & 8.94 & 1.99 & -0.004 \\
\hline
\end{tabular}


Int.J.Curr.Microbiol.App.Sci (2017) 6(6): 3177-3185

\begin{tabular}{|c|c|c|c|c|c|c|c|c|c|c|}
\hline \multirow[t]{2}{*}{$\mathrm{SN}$} & \multirow[t]{2}{*}{ Genotype } & \multicolumn{3}{|c|}{ Grain Yield Per Plant $(\mathrm{g})$} & \multicolumn{3}{|c|}{ Oil content $\%$} & \multicolumn{3}{|c|}{ Protein content $\%$} \\
\hline & & $\mu$ & $\mathrm{b}_{\mathrm{i}}$ & $\mathrm{S}^{2} \mathrm{~d}_{\mathrm{i}}$ & $\mu$ & $b_{i}$ & $\mathrm{~S}^{2} \mathrm{~d}_{\mathrm{i}}$ & $\mu$ & $b_{i}$ & $\mathrm{~S}^{2} \mathrm{~d}_{\mathrm{i}}$ \\
\hline 58 & L10 x T3 & 90.33 & 1.46 & -4.359 & 4.42 & -0.49 & $0.430 * *$ & 7.88 & 2.59 & -0.005 \\
\hline 59 & $\mathrm{~L} 11 \times \mathrm{T} 3$ & 92.76 & 1.67 & 15.070 & 4.61 & 2.91 & $0.045^{* *}$ & 10.12 & 2.61 & 0.007 \\
\hline 60 & $\mathrm{~L} 12 \times \mathrm{T} 3$ & 88.99 & 1.34 & 4.098 & 4.24 & 3.40 & $0.488 * *$ & 7.75 & 1.11 & 0.001 \\
\hline 61 & L13 x T3 & 90.29 & 1.50 & $88.479 * *$ & 4.18 & 10.70 & $0.080 * *$ & 9.71 & 1.88 & 0.005 \\
\hline 62 & $\mathrm{~L} 14 \times \mathrm{T} 3$ & 93.61 & 2.29 & 0.653 & 4.38 & -0.44 & -0.001 & 10.67 & 1.04 & -0.002 \\
\hline 63 & $\mathrm{~L} 15 \times \mathrm{T} 3$ & 85.22 & 1.10 & 2.103 & 4.24 & 0.22 & -0.000 & 8.92 & 1.82 & -0.005 \\
\hline 64 & HQPM-1 & 88.16 & 1.10 & -5.795 & 4.46 & -1.83 & $0.109 * *$ & 9.38 & 3.51 & $0.018 *$ \\
\hline 65 & HQPM-5 & 75.16 & 0.63 & -6.853 & 4.62 & 1.67 & $0.005^{*}$ & 7.59 & 0.58 & -0.004 \\
\hline 66 & Pratap-QPM-1 & 48.44 & 0.37 & -6.495 & 4.00 & 8.54 & $0.091 * *$ & 7.77 & 0.49 & -0.005 \\
\hline \multirow[t]{3}{*}{67} & Vivek- QPM-9 & 94.84 & 2.21 & 15.433 & 4.43 & -0.37 & $0.090^{* *}$ & 8.85 & 0.69 & -0.004 \\
\hline & Mean & 74.58 & & & 4.23 & & & 8.87 & & \\
\hline & SE (b) & 0.21 & & & 2.45 & & & 0.49 & & \\
\hline
\end{tabular}

\begin{tabular}{|c|c|c|c|c|c|c|c|c|c|c|}
\hline \multirow[t]{2}{*}{ SN } & \multirow[t]{2}{*}{ Genotype } & \multicolumn{3}{|c|}{ Starch content (\%) } & \multicolumn{3}{|c|}{ Lysine content (\%) } & \multicolumn{3}{|c|}{ Tryptophan content (\%) } \\
\hline & & $\mu$ & $b_{i}$ & $\mathrm{~S}^{2} \mathrm{~d}_{\mathrm{i}}$ & $\mu$ & $\mathrm{b}_{\mathrm{i}}$ & $\mathrm{S}^{2} \mathrm{~d}_{\mathrm{i}}$ & $\mu$ & $\mathrm{b}_{\mathrm{i}}$ & $\mathrm{S}^{2} \mathrm{~d}_{\mathrm{i}}$ \\
\hline 1 & $\mathrm{~T} 1$ & 61.10 & 3.06 & -0.165 & 2.26 & 1.17 & -0.000 & 0.54 & 0.99 & -0.000 \\
\hline 2 & $\mathrm{~T} 2$ & 66.67 & $2.09 *$ & -0.166 & 1.75 & 1.11 & -0.000 & 0.53 & 0.88 & -0.000 \\
\hline 3 & $\mathrm{~T} 3$ & 66.78 & 3.00 & -0.165 & 1.74 & 0.90 & $0.000 * *$ & 0.56 & 1.41 & 0.000 \\
\hline 4 & L1 & 57.15 & -1.01 & -0.131 & 2.00 & 1.87 & $0.001 * *$ & 0.55 & 0.38 & $0.000^{*}$ \\
\hline 5 & L2 & 62.43 & -0.42 & -0.157 & 2.24 & 1.18 & 0.000 & 0.54 & 1.16 & 0.000 \\
\hline 6 & L3 & 62.26 & 1.50 & -0.153 & 1.97 & 0.51 & $0.000 * *$ & 0.53 & $1.38 *$ & -0.000 \\
\hline 7 & L4 & 67.19 & 0.84 & -0.143 & 0.74 & 0.12 & $0.000 * *$ & 0.57 & 0.91 & 0.000 \\
\hline 8 & L5 & 66.84 & 1.49 & -0.162 & 0.73 & 0.65 & -0.000 & 0.56 & 1.06 & -0.000 \\
\hline 9 & L6 & 62.84 & 1.95 & -0.158 & 1.10 & $1.30 *$ & -0.000 & 0.98 & 1.39 & -0.000 \\
\hline 10 & L7 & 67.49 & 1.93 & -0.156 & 0.87 & 1.57 & 0.000 & 0.52 & 1.06 & -0.000 \\
\hline 11 & L8 & 63.32 & 1.26 & -0.166 & 1.71 & 0.45 & $0.000 *$ & 0.99 & 1.07 & $0.000 *$ \\
\hline 12 & L9 & 56.55 & 1.15 & -0.166 & 1.16 & 1.11 & 0.000 & 0.58 & 0.99 & -0.000 \\
\hline 13 & L10 & 62.57 & 1.46 & -0.123 & 1.88 & 1.69 & 0.000 & 0.64 & 0.26 & $0.000 *$ \\
\hline 14 & L11 & 67.00 & 2.31 & -0.165 & 0.57 & 0.41 & $0.002 * *$ & 0.54 & 1.25 & 0.000 \\
\hline 15 & L12 & 67.37 & $2.11 *$ & -0.166 & 0.77 & 1.31 & $0.000^{*}$ & 0.56 & 0.88 & -0.000 \\
\hline 16 & L13 & 61.25 & $0.99 *$ & -0.166 & 0.71 & 0.65 & 0.000 & 0.54 & $1.45^{*}$ & -0.000 \\
\hline 17 & L14 & 54.44 & 0.76 & -0.165 & 0.76 & 1.63 & 0.000 & 0.57 & 0.90 & $0.000 *$ \\
\hline 18 & L15 & 66.70 & $1.82 *$ & -0.166 & 0.72 & 0.78 & 0.000 & 0.56 & $0.98 *$ & -0.000 \\
\hline 19 & L1 x T1 & 56.18 & 1.61 & -0.154 & 1.20 & 0.84 & 0.000 & 0.60 & $1.54 *$ & -0.000 \\
\hline 20 & $\mathrm{~L} 2 \times \mathrm{T} 1$ & 57.01 & 0.59 & -0.144 & 0.98 & 1.56 & 0.000 & 0.60 & 0.92 & -0.000 \\
\hline 21 & L3 x T1 & 54.34 & $0.72 *$ & -0.166 & 0.99 & 0.78 & 0.000 & 0.67 & 0.91 & 0.000 \\
\hline 22 & $\mathrm{~L} 4 \times \mathrm{T} 1$ & 61.39 & 0.67 & -0.166 & 2.05 & $1.57 *$ & -0.000 & 0.45 & 0.81 & $0.000 * *$ \\
\hline 23 & L5 x T1 & 62.78 & $1.74 *$ & -0.166 & 1.55 & 0.98 & -0.000 & 0.68 & 0.18 & $0.000 *$ \\
\hline 24 & L6 x T1 & 61.64 & 0.63 & -0.165 & 0.83 & 0.65 & 0.000 & 0.57 & $1.53 *$ & -0.000 \\
\hline 25 & $\mathrm{~L} 7 \times \mathrm{T} 1$ & 60.34 & 0.59 & -0.165 & 2.07 & -0.28 & $0.001 * *$ & 0.58 & 1.23 & $0.000 *$ \\
\hline 26 & L8 x T1 & 61.88 & 0.27 & -0.157 & 2.00 & 2.08 & $0.000 *$ & 0.98 & 1.39 & -0.000 \\
\hline 27 & L9 $\times$ T1 & 62.20 & 0.59 & -0.166 & 1.69 & 1.18 & 0.000 & 0.57 & 1.03 & -0.000 \\
\hline 28 & L10 x T1 & 60.98 & 1.65 & -0.166 & 0.79 & 1.31 & 0.000 & 0.55 & $1.54 *$ & -0.000 \\
\hline 29 & L11 x T1 & 61.44 & 0.68 & -0.165 & 0.58 & 0.84 & 0.000 & 0.63 & 1.48 & -0.000 \\
\hline 30 & $\mathrm{~L} 12 \times \mathrm{T} 1$ & 63.75 & 0.75 & -0.166 & 2.10 & 0.92 & -0.000 & 0.55 & 0.89 & -0.000 \\
\hline 31 & $\mathrm{~L} 13 \times \mathrm{T} 1$ & 56.57 & 0.58 & -0.166 & 0.89 & 1.50 & 0.000 & 0.62 & 1.21 & -0.000 \\
\hline 32 & $\mathrm{~L} 14 \times \mathrm{T} 1$ & 61.84 & 0.94 & -0.161 & 1.61 & 1.18 & -0.000 & 0.58 & $1.14^{*}$ & -0.000 \\
\hline 33 & L15 x T1 & 62.08 & 1.03 & -0.166 & 0.93 & 0.85 & 0.000 & 0.59 & 1.04 & -0.000 \\
\hline 34 & $\mathrm{~L} 1 \times \mathrm{T} 2$ & 63.18 & 1.24 & -0.155 & 2.38 & 1.44 & 0.000 & 0.54 & $0.56^{*}$ & -0.000 \\
\hline 35 & $\mathrm{~L} 2 \times \mathrm{T} 2$ & 63.81 & 0.62 & -0.167 & 1.49 & 0.72 & 0.000 & 0.61 & 0.34 & $0.000 *$ \\
\hline 36 & $\mathrm{~L} 3 \times \mathrm{T} 2$ & 61.25 & 0.74 & -0.166 & 1.81 & 0.91 & -0.000 & 0.57 & 0.65 & -0.000 \\
\hline 37 & $\mathrm{~L} 4 \times \mathrm{T} 2$ & 61.43 & 0.55 & -0.165 & 1.64 & 1.18 & 0.000 & 0.67 & 1.08 & -0.000 \\
\hline 38 & $\mathrm{~L} 5 \times \mathrm{T} 2$ & 61.02 & 0.99 & -0.165 & 1.47 & 1.57 & 0.000 & 0.56 & 0.46 & -0.000 \\
\hline 39 & L6 x T2 & 58.58 & 0.81 & -0.163 & 1.09 & 0.92 & 0.000 & 0.57 & 1.89 & 0.000 \\
\hline 40 & $\mathrm{~L} 7 \times \mathrm{T} 2$ & 59.61 & 1.90 & -0.140 & 0.91 & 0.74 & $0.001 * *$ & 0.57 & 1.15 & -0.000 \\
\hline 41 & $\mathrm{~L} 8 \times \mathrm{T} 2$ & 62.27 & 0.12 & -0.160 & 0.74 & 0.78 & -0.000 & 0.57 & 0.87 & -0.000 \\
\hline 42 & L9 $x$ T2 & 61.02 & 1.04 & -0.167 & 0.84 & 1.25 & $0.000^{*}$ & 0.58 & 0.94 & 0.000 \\
\hline 43 & $\mathrm{~L} 10 \times \mathrm{T} 2$ & 56.19 & 2.29 & -0.151 & 1.25 & 1.18 & -0.000 & 0.57 & 1.23 & -0.000 \\
\hline 44 & L11 x T2 & 61.35 & 0.62 & -0.166 & 1.55 & 0.84 & -0.000 & 0.64 & 0.85 & 0.000 \\
\hline 45 & L12 x T2 & 63.36 & 0.54 & -0.166 & 1.66 & 0.71 & -0.000 & 0.63 & 1.25 & 0.000 \\
\hline
\end{tabular}




\begin{tabular}{|c|c|c|c|c|c|c|c|c|c|c|}
\hline \multirow[t]{2}{*}{$\mathrm{SN}$} & \multirow[t]{2}{*}{ Genotype } & \multicolumn{3}{|c|}{ Starch content $(\%)$} & \multicolumn{3}{|c|}{ Lysine content $(\%)$} & \multicolumn{3}{|c|}{ Tryptophan content (\%) } \\
\hline & & $\mu$ & $b_{i}$ & $\mathrm{~S}^{2} \mathrm{~d}_{\mathrm{i}}$ & $\mu$ & $b_{i}$ & $\mathrm{~S}^{2} \mathrm{~d}_{\mathrm{i}}$ & $\mu$ & $b_{i}$ & $\mathrm{~S}^{2} \mathrm{~d}_{\mathrm{i}}$ \\
\hline 46 & $\mathrm{~L} 13 \times \mathrm{T} 2$ & 61.28 & 0.65 & -0.166 & 1.01 & 1.18 & 0.000 & 0.57 & $1.05^{*}$ & -0.000 \\
\hline 47 & $\mathrm{~L} 14 \times \mathrm{T} 2$ & 59.42 & 0.67 & -0.165 & 1.66 & 1.24 & 0.000 & 0.65 & 1.00 & 0.000 \\
\hline 48 & $\mathrm{~L} 15 \times \mathrm{T} 2$ & 64.38 & 0.54 & -0.166 & 0.89 & 0.97 & 0.000 & 0.65 & 1.16 & 0.000 \\
\hline 49 & $\mathrm{~L} 1 \times \mathrm{T} 3$ & 61.56 & 0.83 & -0.165 & 0.68 & 1.51 & 0.000 & 0.55 & 0.92 & -0.000 \\
\hline 50 & $\mathrm{~L} 2 \times \mathrm{T} 3$ & 55.46 & 0.81 & -0.164 & 1.08 & 0.78 & 0.000 & 0.57 & $1.54^{*}$ & -0.000 \\
\hline 51 & L3 x T3 & 61.63 & 0.67 & -0.166 & 0.75 & 0.78 & -0.000 & 0.57 & 0.82 & -0.000 \\
\hline 52 & $\mathrm{~L} 4 \times \mathrm{T} 3$ & 58.28 & -0.91 & -0.146 & 1.00 & 1.24 & -0.000 & 0.59 & 0.65 & -0.000 \\
\hline 53 & L5 x T3 & 61.60 & 0.84 & -0.166 & 1.54 & 1.05 & 0.000 & 0.64 & 0.73 & $0.000 * *$ \\
\hline 54 & L6 x T3 & 57.13 & 0.78 & -0.166 & 0.95 & 0.58 & 0.000 & 0.58 & 1.39 & -0.000 \\
\hline 55 & L7 x T3 & 66.96 & 0.63 & -0.166 & 0.75 & 0.79 & 0.000 & 0.53 & 0.92 & -0.000 \\
\hline 56 & L8 x T3 & 57.50 & 0.70 & -0.167 & 1.17 & 0.78 & -0.000 & 0.62 & 0.77 & 0.000 \\
\hline 57 & L9 $x$ T3 & 60.86 & 0.85 & -0.163 & 1.01 & 1.18 & -0.000 & 0.57 & 1.03 & -0.000 \\
\hline 58 & L10 x T3 & 57.36 & 0.96 & -0.165 & 1.01 & 0.71 & $0.000 * *$ & 0.70 & $1.47 *$ & -0.000 \\
\hline 59 & L11 x T3 & 62.90 & 0.75 & -0.166 & 1.77 & 0.58 & $0.000 *$ & 0.57 & 0.82 & -0.000 \\
\hline 60 & L12 x T3 & 58.34 & $0.65^{*}$ & -0.166 & 0.67 & 0.98 & -0.000 & 0.58 & $0.96^{*}$ & -0.000 \\
\hline 61 & $\mathrm{~L} 13 \times \mathrm{T} 3$ & 60.37 & $0.90^{*}$ & -0.166 & 1.53 & 0.08 & $0.001 * *$ & 0.61 & 0.11 & 0.000 \\
\hline 62 & L14 x T3 & 62.18 & 0.56 & -0.164 & 2.30 & 0.97 & 0.000 & 0.65 & 0.62 & -0.000 \\
\hline 63 & $\mathrm{~L} 15 \times \mathrm{T} 3$ & 61.15 & 0.97 & -0.161 & 0.97 & 0.65 & -0.000 & 0.58 & 1.73 & 0.000 \\
\hline 64 & HQPM-1 & 61.29 & $0.51 *$ & -0.166 & 1.94 & 1.57 & 0.000 & 0.57 & $0.72 *$ & -0.000 \\
\hline 65 & HQPM-5 & 66.92 & 2.12 & -0.165 & 1.86 & 0.78 & -0.000 & 0.55 & 0.88 & -0.000 \\
\hline 66 & Pratap-QPM-1 & 67.13 & $0.70^{*}$ & -0.166 & 1.76 & 1.18 & 0.000 & 0.59 & $0.73 *$ & -0.000 \\
\hline \multirow[t]{3}{*}{67} & Vivek- QPM-9 & 56.30 & $1.04 *$ & -0.166 & 2.00 & 0.84 & 0.000 & 0.60 & 0.48 & -0.000 \\
\hline & Mean & 61.45 & & & 1.33 & & & 0.60 & & \\
\hline & SE (b) & 0.40 & & & 0.30 & & & 0.18 & & \\
\hline
\end{tabular}

*, ** Significant at 5 and 1 per cent respectively.

These hybrids thus showed its suitability and stability under favourable environments. Among the checks, Vivek QPM-9 showed non-significant deviation from regression (S2di) and regression coefficient less than unity (bi <1) with higher mean values as compared to the population mean. It was identified stable and suitable under unfavourable environments. A perusal of data for this character revealed that all 67 genotypes (18 parents, 45 hybrids and 4 checks) showed non-significant deviation from regression (S2di) indicating their predictable behaviour for starch content.

Two parental lines viz., L2 and L4 and fourteen hybrids viz., L6 x T1, L8 x T1, L9 x T1, L12 x T1, L2 x T2, L8 x T2, L12 x T2, L15 x T2, L1 x T3, L3 x T3, L5 x T3, L7 x T3, L11 x T3 and L14 x T3 and check PratapQPM-1 exhibited non-significant deviation from regression (S2di) and regression coefficient less than unity (bi <1) and higher mean values as compared to the population mean, were considered suitable and stable under unfavourable environments for starch content. Nine parental lines viz., L3, L5, L6, L7, L8, L10, L11, L12 and L15 and two testers, namely $\mathrm{T} 2$ and $\mathrm{T} 3$ and three hybrids viz., L5 x T1, L15 x T1 and L1 x T2 and check HQPM-5 exhibited non-significant deviation from regression (S2di) and regression coefficient greater than unity (bi $>1$ ) with higher mean values than the population mean. These genotypes thus showed its suitability and stability under favourable environments. One hybrid L14 x T1 showed non- significant S2di and regression coefficient nearly equal to unity (bi $=1$ ) with higher mean values than the population mean. This hybrid was thus stable and suitable in performance under different environments for starch content. In case of lysine content 53 genotypes (11 parents, 38 hybrids and 4 checks) out of 67 genotypes exhibited non-significant deviation from regression (S2di), indicating their predictable behaviour. Four parents viz., L2, L10, T1 and 
T2 showed non-significant deviation from regression (S2di) and regression coefficient greater than unity (bi $>1$ ) with higher mean values than the population mean. These parents were therefore considered suitable and stable in favourable environments. Three hybrids viz., L2 x T2, L11 x T2 and L12 x T2 and two checks HQPM-5 and Vivek QPM-9 exhibited non-significant deviation from regression (S2di) and regression coefficient less than unity (bi <1) with higher mean values as compared to the population mean, were considered suitable and stable under unfavourable environments. Eight hybrids viz., L4 x T1, L9 x T1, L14 x T1, L1 x T2, L4 $x \mathrm{~T} 2, \mathrm{~L} 5 \times \mathrm{T} 2, \mathrm{~L} 14 \times \mathrm{T} 2$ and L5 x T3 and two other checks namely HQPM-1 and Pratap QPM-1 exhibited non-significant deviation from regression (S2di) and regression coefficient more than unity (bi >1) with higher mean values than the population mean. These hybrids and checks were therefore considered suitable and stable under favourable environments. Four other hybrids viz., L5 x T1, L12 x T1, L3 x T2 and L14 x T3 exhibited non- significant S2di and regression coefficient nearly equal to unity (bi $=1$ ) with higher mean values than the population mean, thereby indicating their suitability and stability under different environments.

Stability parameters for this traits revealed that out of 67 genotypes, 58 genotypes (14 parents, 40 hybrids and 4 checks) exhibited non-significant deviation from regression (S2di), indicating predictable behavior for tryptophan content (Table 2). One parental line L6 and eight hybrids viz., L1 x T1, L8 x T1, L11 x T1, L13 x T1, L4 x T2, L12 x T2, L15 $x$ T2 and L10 x T3 showed nonsignificant deviation from regression (S2di) and regression coefficient greater than unity (bi >1) with higher mean values than the population mean, thereby indicating their suitability and stability under favourable environments. Hybrids L2 x T1, L3 x T1 and L14 x T2 exhibited non-significant deviation from regression (S2di) and regression coefficient nearly equal to unity (bi $=1$ ) with with higher mean values than the population mean. These hybrids were considered stable under different environments. Four hybrids viz., L11 x T2, L8 x T3, L13 x T3 and L14 x T3 and one check Vivek QPM-9 exhibited non-significant deviation from regression (S2di) and regression coefficient less than unity (bi <1) with higher mean values than the population mean. These hybrids and check were therefore considered suitable and stable in unfavourable environments. Similar findings for identification of genotypes for their stability under varying environmental conditions were also reported by Agrwal et al., (2000), Dodiya and Joshi (2003), Nirala and Jha (2003), Abera et al., (2004), Kumar and Singh (2004), Kaundal and Sharma (2006), Javed et al., (2006), Abdulai et al., (2007), Worku and Zelleke (2008) Singh et al., (2009), Lata et al., (2010), Rahman et al., (2010), Nahar et al., (2010), Arulselvi and Selvi (2010), Brar et al., (2010) Nadagoud et al., (2012), Shiri (2013), Nzuve et al., (2013), Kamutando et al., (2013). Anley et al., (2013), Tiwari et al., (2014) and Bisawas et al., (2014).

\section{References}

Abdulai, M.S., Sallah P.Y.K. and Safo, K. O. (2007). Maize grain yield stability analyses in full season lowland maize in Ghana. Int. J. Agric. Biol., 9: 41-45.

Abera, W., Labuschagne, M. T. and Maartnes, H. (2006). Evaluation of maize genotypes using parametric and nonparametric stability estimates. Cereal Res. Commun., 34(2/3): 925-931.

Abera, W., Rensburg, J. B. J-Van., Labuschagne, M. T. and Maartnes, H., (2004). Genotype -environment interactions and yield stability of new 
maize in Ethiopia. South African J. Pl. Soil, 21(4): 251-254.

Admassu, S., Nigussie, M. and Zelleke, H. (2008). Genotype- environment interaction and stability analysis for grain yield of maize (Zea mays L.) in Ethiopia. Asian Journal of Plant Sciences, 7(2):163-169.

Eberhart, S.A. and Russell, W. A. (1966). Stability parameters for comparing varieties. Crop Sci., 6: 36-40.

Kaundal, R. and Sharma, B. K. (2006). Genotype $\times$ environment interaction and stability analysis for yield and other quantitative traits in maize (Zea mays L.) under rainfed and high rainfall valley areas of the sub montane. Res. on Crops, 7(1): 171-180.

Kumar, A. and Singh, N. N. (2004). Stability studies of inbred and single crosses in maize. Ann. Agric. Res., 25: 142-148.

Nadagoud, V.K., Jagadeesha, R.C., Wali, M.C., Kachapur, R.M. (2012). Genetic studies on stability among maize inbredlines. Karnataka J. Agric. Sci., 25 (1): 124-126.
Nahar, K., Ahmed, S., Akanda, M. A. L., Mondal, M. A. A. and Islam, A. A. (2010). Genotype-environment interaction for cob yield and maturity in baby corn (Zea mays L.). Bangladesh J. Agril. Res., 35 (3): 489-496.

Nirala, R. B. P. and Jha, P. B. (2003). Phenotypic stability for fodder traits in maize (Zea mays L.). Range Management and Agroforestry, 24(1): 9-13.

Nzuve, F., Githiri, S., Mukunya, D. M. and Gethi, J. (2013). Analysis of genotype x environment interaction for grain yield in maize hybrids. Journal of Agricultural Science, 5(11): 75-85.

Singh, S. B., Sharma, M. M. and Singh, A. K. (2009). Stability analysis for grain yield and yield-contributing traits in maize (Zea mays) single cross hybrids under mid hills. Indian Journal of Agricultural Sciences, 79 (11):890-896

Tiwari, R., Sharma, A. K. and Kumar, B. (2014). Genotype-environment interaction for yield and its attributes in maize (Zea mays L.). Plant Archives, 14 (2): 841-845.

\section{How to cite this article:}

Baudh Bharti, R.B. Dubey, Arun Kumar, Amit Dadheech and Rohit Kumar Dhobi. 2017. Stability Analysis for Grain Yield and Quality Parameters in QPM (Zea mays L.) Inbred Line Crosses. Int.J.Curr.Microbiol.App.Sci. 6(6): 3177-3185. doi: https://doi.org/10.20546/ijcmas.2017.606.374 